\title{
The Relation of the Neutral Zone to the Crest of the Residual Alveolar Ridge in Completely Edentulous Subjects - A Clinico-Radiological Study
}

\author{
Salil Pawah ${ }^{1}$, Amit Gupta², Bhanu Madan ${ }^{3}$
}

\begin{abstract}
A Clinico-Radiological Study was conducted to establish a set relation between crest of residual alveolar ridge and neutral zone so that a uniform pattern could be followed for teeth setting. Thirty healthy edentulous patients with class - I ridge relation and who had not undergone any major oral surgical procedure was included in this study. Patients were divided into two groups, 1, who were edentulous from six months to two year and group 2, who were edentulous from two or more years. Fifteen patients were in each group. Results showed that in group 1 patients, neutral zone lied lingual to or on crest of alveolar ridge and in group 2 patients, neutral zone shifted to buccal side. Therefore, by studying the pattern of shift of neutral zone to buccal side we should come out with a reasonable plan for placing our teeth looking at duration of edentulousness.
\end{abstract}

Keywords: Alveolar Ridge; Neutral Zone; Completely edentulous

${ }^{1}$ MDS

Professor

Department of Prosthodontics

Sudha Rustagi College of

Dental Sciences and Research

Faridabad, Haryana (INDIA)

${ }^{2} \mathrm{MDS}$

Professor

Department of Prosthodontics

Sudha Rustagi College of

Dental Sciences and Research

Faridabad, Haryana (INDIA)

${ }^{3} \mathrm{MDS}$

Professor

Department of Prosthodontics

Sudha Rustagi College of

Dental Sciences and Research

Faridabad, Haryana (INDIA)

\section{INTRODUCTION}

T oss of teeth leaves us with multifactorial changes occurring in 1 the mouth in the form of resorption of alveolar ridge, tongue expansion, laxity of muscles of cheek and face which are unable to counteract tongue forces towards buccal aspect. Hence, leaving crest of residual alveolar ridge, is not an ideal foundation for placement of artificial teeth (1).

Many edentulous people can experience difficulty in carrying out functional activities when wearing complete dentures. This may be due to a number of factors, one of which is the ability to control the dentures effectively during mastication and speech. For this reason, it is critical when constructing complete dentures to incorporate features in their design which will aid stability in function(2). Usually, the challenges are greater in constructing prosthesis to replace the missing mandibular teeth; therefore, neutral zone concept becomes important.
Successful treatment of patients with complete dentures depends mainly upon the proper positioning of the teeth on the denture foundation. Along with vertical height changes, resorption of bone brings about changes in bucco-lingual direction, of residual alveolar ridge. Generally, loss of bone occurs from the lingual plate of mandibular arch and buccal aspects of maxillary arch. This allows more space for tongue movement and hence tongue enlarges over the years. This results in exertion of force more towards buccal and labial sides. Cheeks and lips may not respond in the same fashion due to loss of tonicity of muscles, with advancing age. Therefore neutral zone may not lie at the place where it was, when teeth were present. In 1933, Sir E. Wilfred Fish(3) introduced the concept of neutral zone and gave ideas of placing teeth in this zone. Neutral zone was defined as "that area in the mouth where the forces of tongue pressing outward are neutralized by forces of cheeks and lips pressing inward". This concept was followed and prescribed by many since then for e.g.: Russel (1959)(4) who termed it as 


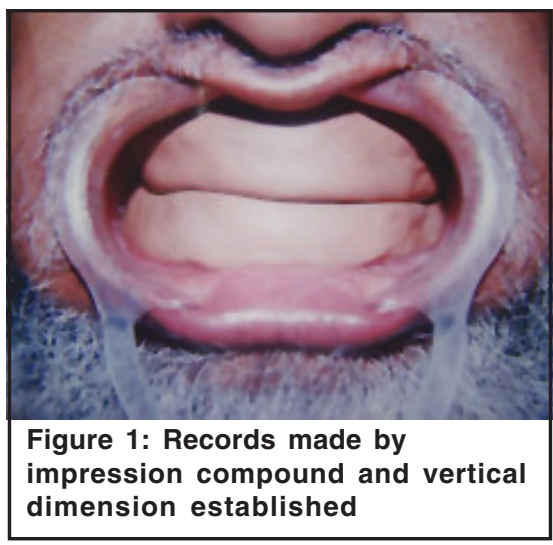

"Reciprocal space", Robert (1960)(5) called it the "Potential space", Heath (1970) (6) as "Denture space" Bates (1984) as "Reciprocal zone", Mathew (1961) (7) as "Zone of minimum conflict" and Fenn (1986) (8) termed it "Zone of neutral muscular forces".

There were numerous advantages of neutral zone dentures such as: Better comfort and speech, more room for tongue, Easy to adjust for the patients, more in harmony with surrounding perioral musculature. However, some authors contraindicated this concept stating, Masticatory efficiency inadequate, unnecessary torque forces getting generated, less stable due to lateral stresses generated. Over the past forty years, numerous articles which have touched upon or related to what we have termed the "neutral-zone concept" have appeared in the literature. Unfortunately, the profession generally has ignored these ideas, and consequently the principles are frequently bypassed in denture construction. Complete dentures are primarily mechanical devices, but since they

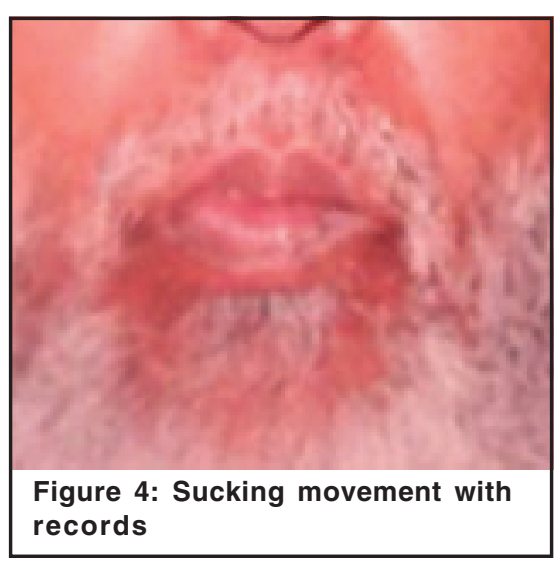

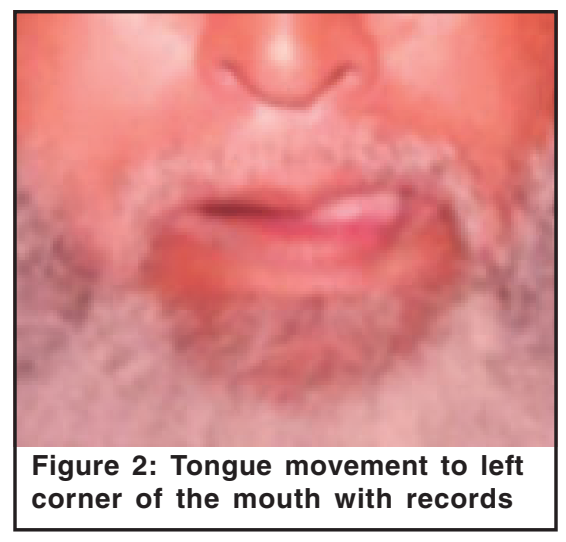

function in the oral cavity, they must be fashioned so that they are in harmony with normal neuromuscular function. All oral functions such as, speech, mastication, swallowing, smiling and laughing involve the synergistic action of the tongue, lips, cheek and floor of the mouth which are very complex and highly individual. Failure to recognize the cardinal importance of tooth position and flange form and contour often results in dentures which are unstable and unsatisfactory, even though they were skillfully designed and expertly constructed. The coordination of complete dentures with neuromuscular function is the foundation for successful, stable dentures(9).

Since a lot of controversial opinions have been expressed about benefits of conventional and neutral zone philosophy and placement of artificial teeth, it was essential to investigate and research into, by a method economically viable and convenient to provide not only functionally but psychologically satisfying dentures to the patients.

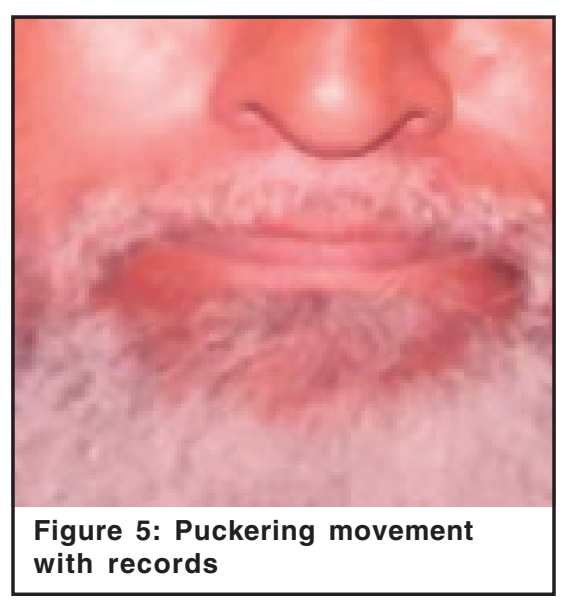

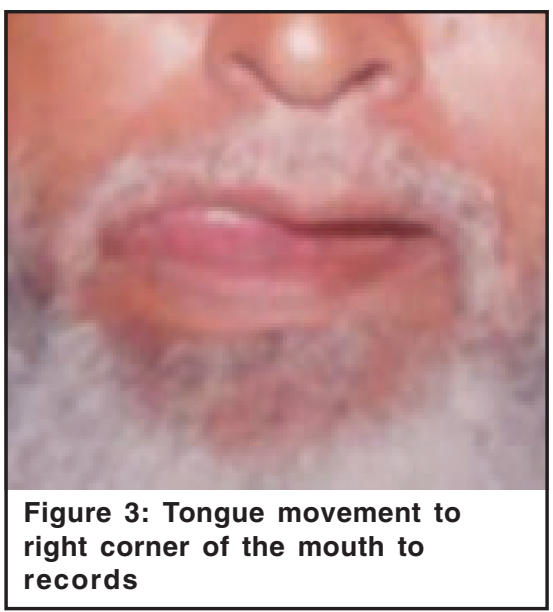

\section{MATERIALS AND METHOD}

Thirty healthy edentulous patients, who agreed to participate in this study, were included in this study. These patients were having class I ridge relation and had not undergone any major oral surgical procedure. Patients were divided into 2 groups, One, who were edentulous from 6 months to 2 years and second, who were edentulous from 2 or more years. Division was equal i.e. 15 patients were in each group. Neutral zone recording was done with each patient at established vertical dimension (Figure 1).

Primary impressions were made in impression compound and the casts poured in dental plaster. Custom trays were prepared on primary cast in autopolymerizing resin by dough method. These were reduced $2 \mathrm{~mm}$ for muscle trimming with tracing sticks of low fusing impression compound. Border moulding was done to proper extension and secondary impression was made in Zinc oxide Eugenol paste. Secondary casts were poured with dental stone. After obtaining

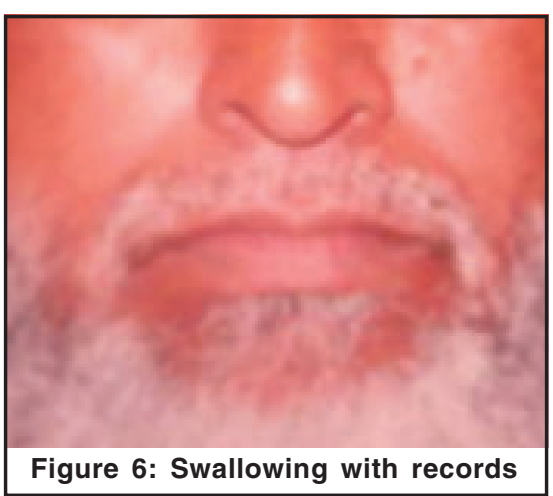




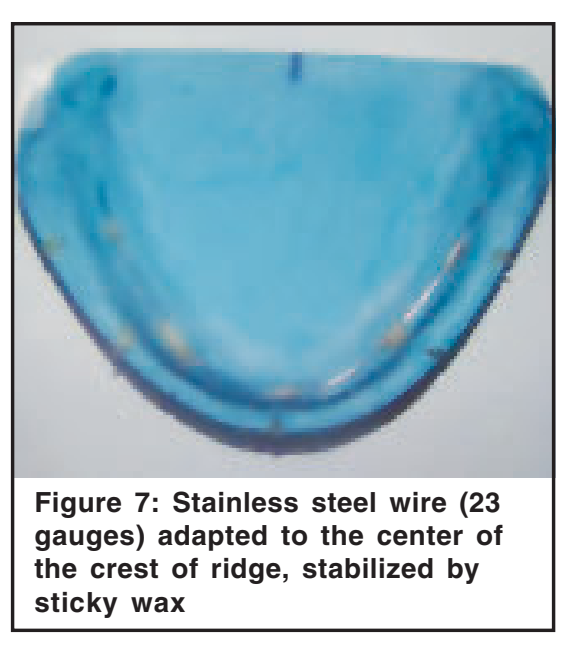

the secondary cast, these casts were duplicated in Agar-Agar in duplicating machine. Two pairs of duplicating cast were made - One pair of duplicating casts were used for making permanent record bases in Heat cure acrylic resin. The bases were tried in patients' mouth and were checked for comfort, retention, stability and extension. Impression compound was softened in a $65^{\circ} \mathrm{C}$ water bath. The softened compound was attached to the crest of mandibular recording base. A small amount of the compound was manipulated along the labial, buccal and lingual slopes. Attached roll of compound was reheated in water bath and was carried into the patients' mouth. With record bases seated in to the patients mouth and fingers out of patients mouth, patient was asked to: Protrude the tongue in the same pattern for each patient, Touch corner of lips, Sucking movement, Puckering, Swallowing (Figure 2-6). This was carried till established Vertical Dimension was attained. After compound cooled, the record base with compound rim was removed.

The crest of the ridge of $2^{\text {nd }}$ pair of casts was trimmed $1 \mathrm{~mm}$ with round bur. A 23 gauge stainless steel wire was adapted along the centre of the alveolar ridge (Figure 7). This wire was stabilized by sticky wax. The centre of the buccolingual width of each compound was marked along its length. A 30 gauge stainless steel wire was stabilized over the centre of each occlusal rim (Figure 8). Record base was positioned back on the facial cast., Before that, cast was marked with radiopaque marker on left molar, left

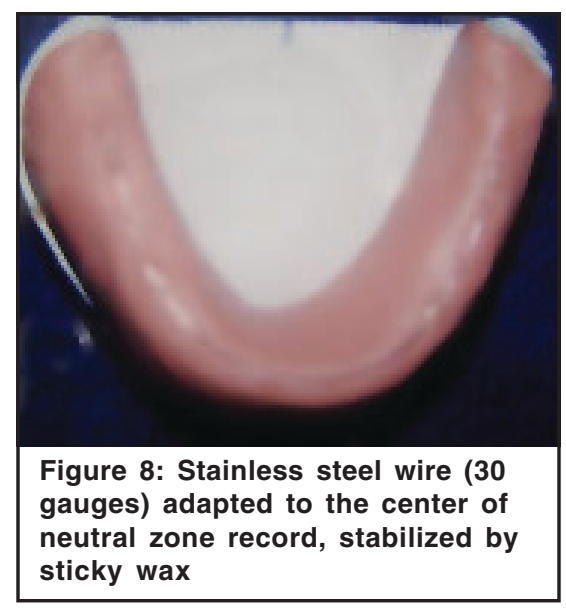

premolar, anterior right premolar and right molar on buccal aspect. Now, an occlusal view radiograph was obtained of each record base and its casts using specified radiographs (Figure 9). The object to source distance was 6 inches and central ray was directed at the centre of the cast. Exposure parameters used were $70 \mathrm{Kvp}, 8 \mathrm{~mA}$ current, 1.5 seconds was exposure time for each radiograph. All the films were developed. Each film was viewed, where thicker wire indicated crest of the ridge (centre) and thinner one the neutral zone (centre). Relationships between images were studied in bucco-lingual direction. Where two images coincided a zero score was assigned, buccal and labial locations of the Neutral Zone with respect to the ridge were assigned a positive value. Lingual locations of the Neutral Zone with respect to the ridge were assigned a negative value. 98 measurements were made with a vernier caliper to an accuracy of $0.01 \mathrm{~mm}$.

\section{RESULTS}

The patients were classified according to their period of edentulousness into 2 groups- $I^{\text {st }}$ group edentulous from $1 / 2$ to 2 years. All the measurements were in millimeter. The location of neutral zone with respect to center of crest of residual alveolar ridge was studied at 5 different locations i.e. Right Molar, Right Premolar, Anterior, Left Premolar and Left Molar (Figure 7,9).

Observations were made that in this group of patients of $1 / 2$ to 2 years of edentulousness, depicted that 59 of 75

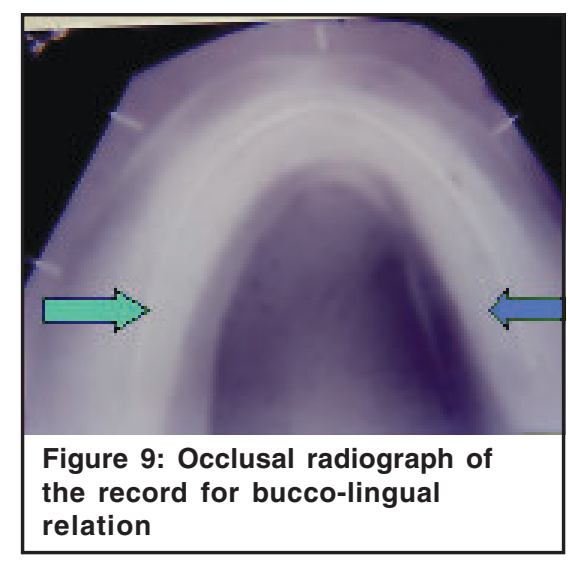

locations studied $(78.66 \%)$ neutral zone was found lying lingual alveolar ridge. 12 out of 75 locations it coincided with center of crest of residual alveolar ridge (16\%). 4 out of 75 locations it lied buccal to center of crest of residual alveolar ridge (5.33\%). Summing up together the results of above group we can say that neutral zone lied lingual to center of crest of ridge and had significant variations from center of residual alveolar ridge. In the second group with 15 subjects edentulous from 2 or above years, 39 out of 75 noted $(52 \%)$ neutral zone lied buccal to the center of the crest of the ridge, 14 out of 75 locations (18.66\%) neutral zone coincided with center of the crest of residual alveolar ridge, 22 out of 75 locations $(29.33 \%)$ neutral zone lied lingual to the center of the crest of residual alveolar ridge, which meant that there is tremendous buccal shift of neutral zone with advancement of the duration of edentulousness.

\section{DISCUSSION}

Present study is focused on buccolingual variation of bone and thus, neutral zone with advancement in age and subsequently arranging teeth accordingly, so as to provide a stable complete denture. Thus, the teeth should be arranged in such a position that the forces from the tongue are equalized by the forces of the lips and cheeks. Teeth should not be stationed away from crest so much that undue lateral stresses get generated.

Dr. Musyken (1961) (10) estimated that if the size of mandibular teeth is too large or posterior teeth are set $1 \mathrm{~mm}$ lingually, the tongue is deprived of approximately 1000 
cubic $\mathrm{mm}$ of functional space. This can force the tongue into an abnormal retracted position which would adversely affect speech. Rinaldi et al. (1963) (11) gave similar suggestion.

Observations of the study suggested that neutral zone shifted to buccal side as we can make out by decrease in lingual locations in $2^{\text {nd }}$ group and increase in buccal locations. These observations were in accordance with Rajek and Abdalla (1981) (12). Observations also stated that blindly positioning teeth on the crest of the ridge does not aid retention, support and stability but can cause cramping of tongue, inadequate cheek support and an uncomfortable denture. Also, placing teeth too buccally as suggested by certain neutral zone readings ( $+5 \mathrm{~mm}$ etc.) can cause midline fractures due to lateral stresses and inadequate relief to midpalatine raphae, development of cheek bite, so we should be a little cautious in teeth placement. However, there was a lot of controversy on masticatory efficiency of the denture because buccal placement of teeth as suggested by this study for $2^{\text {nd }}$ group of patients, may cause undue lateral stresses and inadequate support from bone as suggested by Weinberg (1957) (13). Present study gives us an easy and inexpensive way to determine the relationship between crest of alveolar ridge and neutral zone, incorporating it into practice will be a great aid to our patient.

\section{CONCLUSION}

The results indicated that neutral zone shifted towards buccal and labial side with increase in duration of edentulism. Also, placement of teeth should not always be on the crest of the ridge because crest of residual alveolar ridge does not coincide with the neutral zone as it resorbs differently anteriorly and posteriorly. Neuromuscular control plays an important role in determining the placement of teeth for a comfortable denture. Combination of all these factors should be taken into account for placement of teeth for comfortable prosthesis and it was attempted by a simple clinical method to incorporate all these together.

\section{REFERENCES}

1. Fahmi FM. The position of the neutral zone in relation to the alveolar ridge. $J$ Prosthet Dent 1992;67:805-09.
2. Scott BJ, Hunter RV. Creating complete dentures that are stable in function. Dent Update 2008;35(4):259-62,265-67.

3. Fish EW. Principles of full denture prosthesis. $5^{\text {th }}$ edn. Staples Press; 1952.

4. Russel AF. The reciprocal lower complete denture. J Prosthet Dent 1959;9(2):18090.

5. Roberts AF. The effect of outline and form upon denture stability and retention. Dent Clin North Am 1960;293-303.

6. Heath R. A study of the morphology of the denture space. Dent Pract 1970;21: 109.

7. Mathew $M H$, Wain $A E$, et al. The full denture problems. Br Dent J 1961;61(5): 401-18.

8. Fenn RB, Liddelow KP, Gimson AP. Clinical Dental Prosthesis. CBC publishers and distributers 1986.

9. Victor BE, Frank SJ. The neutral zone in complete dentures. J Prosthet Dent 2006;95(2):93-101.

10. Musyskens Dr. Abstract from: Wright CR, Swartz WH, Godwin WS. Mandibular denture stability. Ann Arbor. Mich, 1961, The Overbeck co.

11. Rinaldi P, Sharry J. Tongue force and fatigue in adults. J Prosthet Dent 1963; 13(5):857-65.

12. Razek MA, Abdalla F. The dimensional study of the neutral zone at different occlusal vertical heights. J Prosthet Dent 1981;46(5):482-89.

13. Weinberg LA. Tooth retention to the denture base foundation. J Prosthet Dent 1957;8:398-405. 